\title{
Freeze-casting applied to ceramic materials: a short review of the influence of processing parameters
}

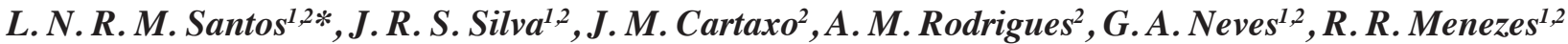 \\ ${ }^{1}$ Universidade Federal de Campina Grande, Programa de Pós-Graduação em Ciência e \\ Engenharia de Materiais, Av. Aprígio Veloso 882, 58429-900, Campina Grande, PB, Brazil \\ ${ }^{2}$ Universidade Federal de Campina Grande, Centro de Ciência e Tecnologia, Campina Grande, Unidade \\ Acadêmica de Engenharia de Materiais, Campina Grande, PB, Brazil
}

\begin{abstract}
In recent years there is a high interest in the freeze-casting process because it is a simple, economical, and environmentally friendly method for obtaining highly porous materials. Most of the materials obtained by this technique have directional pore structure and anisotropic properties. However, a wide range of pore size and morphology can be obtained by the control of processing parameters, such as solvent type, solids concentration, particle size, freezing rate, and types of additives. The varied morphologies permit the application of freeze-casted materials in various technological applications. However, despite the high amount of studies about freeze-casting of ceramic materials in the last years, review articles addressing the influence of processing parameters on the pore characteristics of obtained ceramics are still scanty. Therefore, this review addresses the influence of freeze-casting process parameters on the pore characteristics of the ceramic materials.
\end{abstract}

Keywords: freeze-casting, pore morphology, freezing rate, solvent, ceramic.

\section{INTRODUCTION}

Porous ceramic materials have taken the attention of the scientific community and are themes of several theoretical and experimental studies in the last years due to their wide range of commercial applications [1]. As a consequence, new processing methods to obtain these kinds of materials are discovered as well as the enhancement of existing methods. As a consequence, it is possible to control the concentration and morphology of the pores aiming to improve desired properties [2]. Among the most approached processing methods, stand out the replica methods, direct foaming, sacrificing template, gel-casting, and freeze-casting [3-6].

The freeze-casting method, also known as freezedrying, has high visibility for presenting the advantages of being a simple, inexpensive, and environmentally friendly method capable of producing ceramic pieces with high porosity (25\% to $90 \%$ ) and with the possibility to control the morphology of these bodies, compared to the methods mentioned above [7, 8]. In addition to being a fast and low-cost processing technique, it has a low environmental impact since it does not require porogenic agents that can release toxic gases during burning as in the replication and sacrifice template methods. Also, the stabilizing agents, such as some toxic surfactants used in the direct foam method, are not employed in the method discussed in this review article. Besides, the freeze-casting method allows high control of pore morphology, making it possible to obtain morphologies ranging from lamellar to cellular, unlike other methods that

*larriseanathiere@hotmail.com

Dhttps://orcid.org/0000-0003-3169-5565 are restricted to the morphology of the porogenic agent used. This method was first described in 1954 by Maxwell et al. [9] in a technical memorandum, which deals with an investigation to obtain bodies of complex-shapes from the processing of refractory powders. However, this initial study used the freeze-casting method as a tool to manufacture dense bodies rather than high-porosity pieces. Later, Tong et al. $[10,11]$ applied the method to a water-soluble polymer, with the addition of agar gels on the suspension, for the synthesis of highly porous materials with well-controlled cellular architecture. Nevertheless, the pioneering studies to obtain macroporous ceramics bodies from the freeze-casting method were published only in 2001 by Fukasawa et al. [12, 13], who studied the production of porous alumina ceramics. Since then, several studies have been carried out to improve the understanding of the freeze-casting method. In Fig. 1, it is possible to see that there has been significant growth in recent years involving research to develop porous systems using freeze-casting. Fukushima's research group has conducted several studies [2,14-21] focused on the manufacture of high porosity materials through freeze-casting. In 2018, Tang et al. [22-26] also turned their researches to the method, and in 2019, several authors [27-34] reported studies involving freeze-casting to obtain materials with high porosity and morphological pore control. Therefore, this scientific field is in continuous growth, and studies that improve the method are published even today.

The freeze-casting method has been applied in various types of materials, such as ceramics, metals, clays, and polymers, and also to nanomaterials and composites [32-37]. Regardless of the material processed, the main focuses of the studies currently refer to the understanding of the influence 

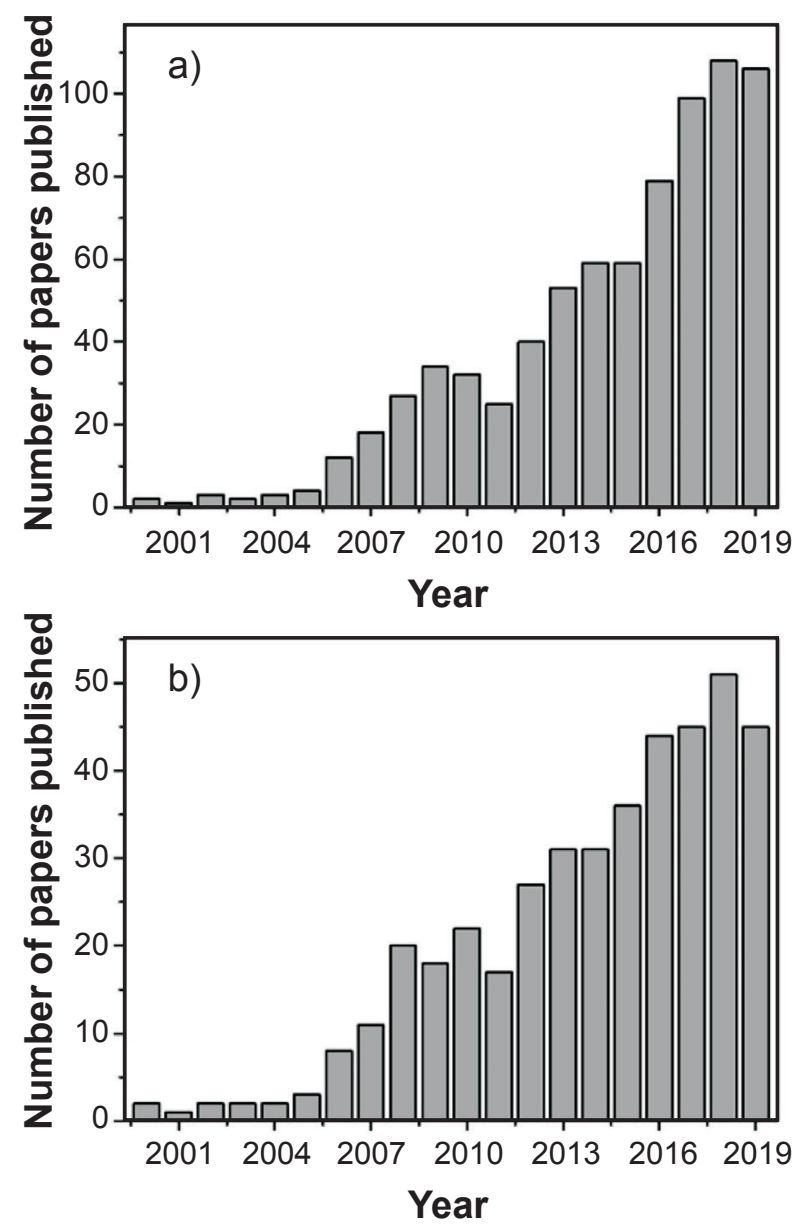

Figure 1: Number of papers published over the past years regarding the freeze-casting process (a), and the number of papers focused on the preparation of ceramic materials by freeze-casting (b), from 2000 to 2019 based on Web of Science Core Collection.

of process parameters, such as solid concentration (solid/ liquid ratio), type and size of the solid particles, solvent type, freezing temperature and direction, and freezing rate, on the final characteristics of the pieces manufactured, such as concentration, size, and morphology of the pores. The pore morphology and size are critical factors that frequently determine the materials applications [38]; therefore, the understanding of the influence of processing parameters on these characteristics becomes extremely important for proper application of the method and success in the production of porous bodies. Most published reviews of this technique emphasize the importance of process parameters; however, they do not generally cover how each parameter influences the final pore morphology. Thus, this paper aims to perform a short review of the influence of freezecasting process parameters on the pore characteristics of the obtained materials.

\section{FREEZE-CASTING PROCESS STEPS}

Freeze-casting is a relatively simple process that has several advantages, such as easy implementation, low-cost, environmentally friendly, and applicable to a wide range of materials [36]. However, understanding the influence of process parameters on the morphology of the manufactured pieces makes it quite complex. When the freeze-casting method is applied to obtain ceramic pieces from powder particles, this method consists basically of four steps: 1) preparation of the aqueous or non-aqueous ceramic suspension; 2) molding and freezing of this suspension; 3) sublimation of the solidified solvent, and 4) sintering. The last step consolidates the ceramic walls while maintaining the pore structure and preserves a replica of the solidified structure of the solvent [7, 12, 13, 38-40]. Fig. 2 shows a schematic of these steps. On the preparation of the suspension (step 1), properties of the solid particle, such as concentration, size, shape, and chemical characteristics, strongly affect the morphology and microstructure of the final piece [30]. Some of these factors are discussed in detail later. In general, to obtain homogeneous macroporous pieces, the suspensions prepared must contain stable, well-dispersed solid particles $[8,41]$. Also, as the process is based on a wet route, the suspension should contain a low concentration of the solid particles to obtain pieces with high porosity. The choice of the solvent is also of great importance, as the frozen solvent acts as a porogenic agent to produce porous pieces [42]. This review presents a specific topic about the influence of different types of solvents on the final morphology, where, in general, the way the solvent is frozen and its characteristics determine the final material's microstructure.

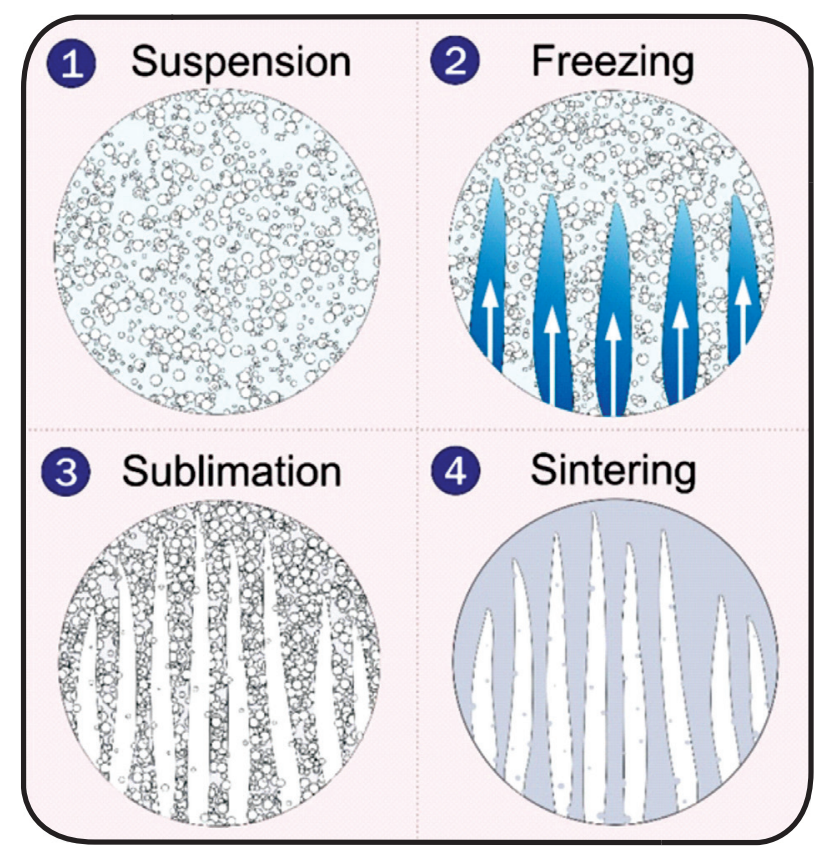

Figure 2: Schematic of the steps of the freeze-casting method.

The freezing step (step 2) is considered the most crucial phase of the process. In this step, there is a growth of the solidified crystal, which gives rise to the porosity after the sublimation of the frozen solvent. This phenomenon has an origin in the low solubility of the solid phase in the solvent, which ensures its segregation during solidification [41] and causes any solid originally dispersed in the aqueous medium 
to be expelled to the boundaries between adjacent crystals [43]. Thus, during the freezing step, solvent crystals grow, and solute molecules are excluded from the frozen solvent until the piece is entirely frozen [42]. When a colloidal suspension is initially cooled from the surface that is in direct contact with the freezing medium, an initial transient regime occurs systematically in the absence of crystal seeds. After the initial transient regime, the crystals reach a steady growth regime, where their growth kinetics and morphology are substantially constant. Initial nucleation and growth result in a structural gradient close to the cooling surface, corresponding to the progressive selection of the stable crystal structure. The pore size and density of the materials vary within this zone. Nucleation can also be controlled to provide additional adjustments to the structure, producing complex and ordered crystal patterns [41]. The crystal growth velocity, crystal morphology, angle between crystal growth direction, and temperature gradient are fundamental factors during freezing, which determine the particle redistribution [7] and, consequently, the final pore morphology. In general, the formation of porous microstructures after freezing of a colloidal suspension depends on the rejection of the particles by the advanced solidification front. The breaking of the solidification front to a non-flat morphology, the concentration, and retention of particles between solidified crystals induce the formation of high aspect ratio ice platelets or dendrites that swallow or reject particles depending on the size and velocity of the crystal growth front $[8,38]$. An important observation is that during the freezing of such suspensions, there is a critical particle size above which the suspended particles are captured by the moving freezing front [44]. The formation of small or large ice crystals after freezing is also related to the concentration of solute in the suspension and the size of the solute [43].

On the third step of the process (Fig.2), the frozen material is sublimated, and pores are developed in pieces; therefore, the porosity of the materials came from the replicate of the original frozen phase structure. As solidification is often directional, channels on the macrostructure of the pieces are developed due to the anisotropic morphology [1]. After the sublimation, there is one of the main problems with the freeze-casting; the pieces resultant become very fragile and difficult to handle. In this way, additional efforts are underway to improve the strength of green bodies [45]. One way is the addition of binders, such as polyvinyl alcohol (PVA), which is a water-soluble binder capable of improving the mechanical stability of green bodies. The last step of the freeze-casting process (Fig. 2) is the sintering of the pieces, which promotes wall consolidation, densification, and improves strength. The final pore content depends on many additional parameters that affect particle packing between the solvent crystals and can be adjusted by varying the particle content, particle size distribution and morphology, the nature of the solvent, its viscosity and surface tension, and the presence of various additives in the suspension $[1,46]$.

\section{MICROSTRUCTURE}

The adaptability of the freeze-casting method makes it capable of fabricating materials with directional or homogeneous microstructures (anisotropic or isotropic) such as dense, cellular, and lamellar microstructures. Although the method can be applied to the production of dense materials, the purpose of the technique today is to create materials with adjustable porous microstructures $[8,47]$. In addition to the versatility of freeze-casting microstructures, the method also enables materials with a porosity of up to $90 \%$ with interconnected open pores and pore size gradient to be produced, resulting in complex shapes and effectively eliminating capillary forces and pore collapse [7, 44, 48]. Fig. 3 shows the types of pore morphologies observed in the literature, which are obtained by freeze-casting; these microstructures are modified by varying the process parameters.

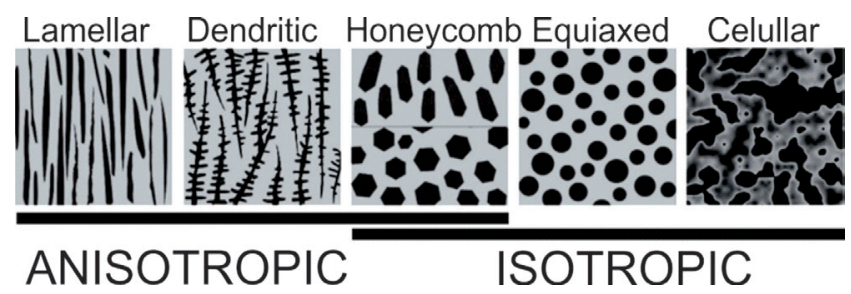

Figure 3: Types of anisotropic and isotropic microstructures that can be obtained by freeze-casting.

The development of anisotropic microstructures, such as lamellar and dendritic, are favored by high-temperature gradients or when the expelled particles are arranged in a specific way [38, 49]. The formation of the lamellar pores with large dimensions along the preferred directions of crystal growth depends on increasing the temperature gradient while decreasing the impediment of the solid particle. On the other hand, directional growth of crystals is inhibited, and homogeneous (cellular) microstructures with interconnected pores can be obtained after sublimation when freezing conditions are controlled to achieve uniform temperature and freezing rate throughout the sample, and particle size is suitably chosen to compensate for the expulsion of particles from the freezing front [50]. So, the homogeneous microstructure is obtained under controlled conditions, in which a minimum temperature gradient is allowed throughout the pieces with low expulsion of the particle. The temperature gradient depends on the characteristics of the pieces such as volume, shape, thermal conductivity, and freezing rate of the sample, while the particle expulsion impediment is influenced by the viscosity of the suspension and the size and shape of the solid particles [50].

\section{EFFECTS OF PROCESS PARAMETERS ON PORE MORPHOLOGY}

In the freeze-casting method, the process parameters, such as solid filler, particle size, solvent, freezing temperature, 
freezing rate, freezing direction, and additives, are essential to control the final microstructure of the pores, and therefore, micro and macro-aspects of the pieces manufactured, i.e., microstructure and sophisticate design at various length scales $[1,43,44,51,52]$. The understanding of how these process parameters influence the final microstructure of the pores is not trivial since the freezing of the concentrated colloidal suspensions is a complex physical process in which many kinetics and thermodynamics parameters are involved [53]. Therefore, review articles, handbooks, and empirical works are relevant information sources that identify state of the art and point to new technological and scientific discoveries.

\section{Amount of solid in the suspension}

The macro and micro-aspects of the pieces manufactured by the freeze-casting method are strongly dependent on the amount of solids in the suspension [1], and the final porosity of the produced pieces is directly related to the ratio volume of solid/volume of solvent in the suspension. The optimum amount of solids and, consequently, the final porosity are dictated by the final application of the piece. For example, pieces with lower porosity are frequently manufactured from suspension with a higher content of solid and lower solvent content. These pieces are used in an application where strength is a critical point. Additionally, for the cases where the water is the solvent, low water concentrations entail that the expansion of the ice crystals does not significantly affect the process; therefore, dense pieces are manufactured. Suspension with a high content of the solid fillers is not suitable for applications requiring high porosity, above $70 \%$. On the other hand, suspension with low content of solid produces green bodies with low strength, difficult to handle, and sintered bodies with relatively low strength, which may limit their applicability [1, 50,54].

Usually, the solid amount is used in a range of 10 to 50 vol\% of the entire suspension. A lower concentration of solids in the suspension would be a problematic situation since the manufactured pieces may have thin walls and low mechanical integrity. On the other hand, a higher concentration of solid means less concentration of solvent in the slurry, and the pieces obtained from this kind of suspension may have few or no connected pores since the crystal growth of the solvent is negatively affected [40, 55]. For pieces manufactured from suspension with a low concentration of the solid (in the range of 10 to $50 \mathrm{vol} \%$ ), it is common the formation of pores larger than $100 \mu \mathrm{m}$, which can limit their application as they have low mechanical properties. However, it is essential to emphasize that the primary influence of variation of the solid concentration in the suspension is on the total porosity of the piece [56]. Fig. 4 shows experimental data acquired from the literature showing the relationship between the porosity and the concentration of solid fillers for ceramic materials manufactured from the freeze-casting method. The rise in the concentration of solids decreases the porosity of the ceramic pieces manufactured by the freeze-casting method (Fig. 4). Most authors used the concentration of the solid between 10 and $60 \mathrm{vol} \%$, focusing on concentrations between 20 and $40 \mathrm{vol} \%$. Indeed, ceramics pieces obtained from these suspensions usually present high porosity (usually above $50 \mathrm{vol} \%$ ) and adequate mechanical resistance to various application areas.

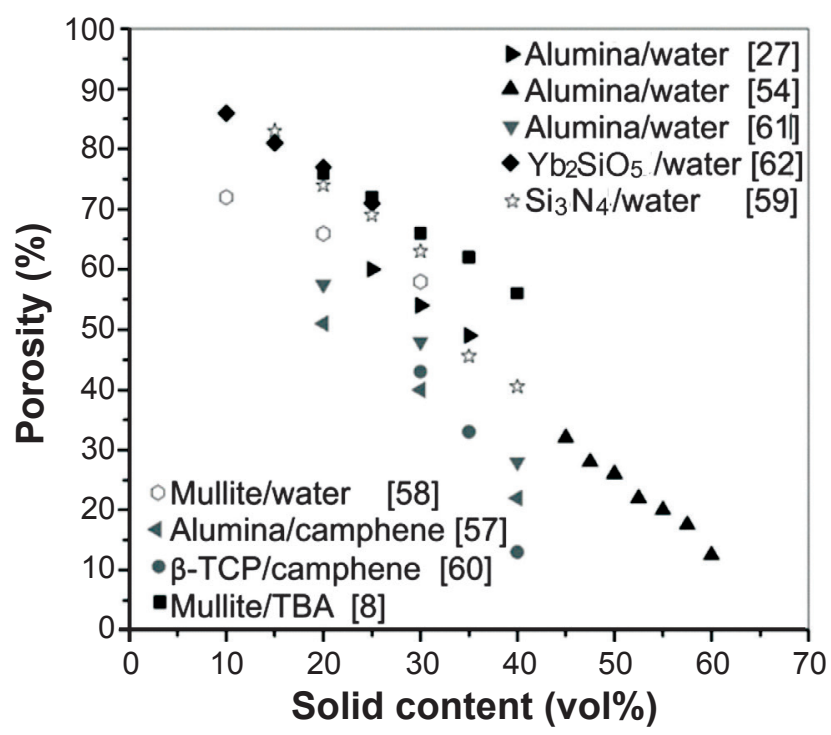

Figure 4: Relationship between porosity and the amount of solids in the suspension for freeze-cast ceramics [8, 27, 54, 57-62].

Solute concentration also plays an important role in the final morphology of the microstructure. Eventually, very high solute concentrations inhibit the formation of solvent crystals and thus the formation of microstructure with microchannels. Moreover, lamellar structure and interconnected pores may be lost in ceramics pieces manufactured from suspension with a high concentration of solid ( $>80 \%$ by weight) [1]. On the other hand, very low concentrations may produce high asymmetric microstructures composed of microchannels but with low strength [43]. When the freeze-casting method is employed to obtain ceramic pieces from suspension with a concentration of solid less than $30 \%$, binder additives are generally required due to mechanical handling requirements. Here is essential to emphasize that the lamellar structure does not seem to be affected by the decrease in the concentration of solid fillers. The literature $[43,56]$ reports that the amount of solid also influences the pore size. In this way, suspensions with a high concentration of solid and/or large particle sizes produce small crystals of the solvent, while suspension with a low concentration of solid fillers and/or small particle sizes favor the formation of the macroporous.

\section{Particle size}

The particle size has significant importance on the microstructure of the pieces manufactured from the freezecasting method. Indeed, the segregation and redistribution 
of particles by a solid/liquid interface is strongly dependent on the particle size, and above the critical particle size, the encapsulation of the particles occurs through a mobile interface [41, 63]. The mobile interface first encapsulates large particles. As a result, a structural gradient appears in the intercrystalline space, in which large and small particles segregate in the outer and inner regions, respectively. On the freeze-casting method, the appearance of new nuclei from the solvent is a heterogeneous process, in which the particles in the suspension act as the nucleation sites. Therefore, smaller particle sizes provide more nucleation sites, and as a consequence, it is expected that the piece manufactured will have a higher concentration of the small pores [50]. Several studies analyze this relationship between particle size and final pore size, as can be seen in Fig. 5. However, based on literature data, which used a range of particle size from $\sim 0.5$ to $15 \mu \mathrm{m}$, the decrease in pore size with the particle size is not easily observed (Fig. 5). This can be attributed to the use of small particles in all data, which produced small pores in all analyzed studies. Moreover, other process parameters such as solvent type, amount of additive, freezing temperature, and solid content strongly affect the pore size. This can hide the influence of the particle size on the final pore size.

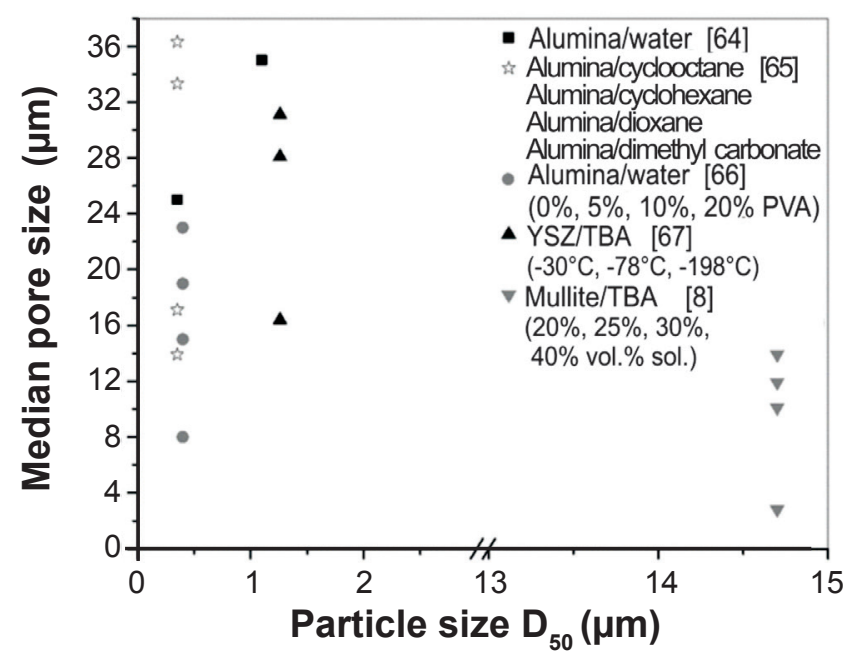

Figure 5: Literature data on particle size and pore size of ceramic materials obtained by freeze-casting [8, 64-67].

Larger particle sizes decrease the critical speed of the freezing front [38]. Conceptually, large particles have more surface area and higher surface energy (per particle) when suspended in the liquid phase, which influences the interaction of the particles with the freezing front. Thus, suspensions with large particles have a higher degree of constitutional supercooling, and the pieces manufactured from them have a macroporous microstructure [30]. A study [46] shows a proportional relationship between particle size and interface velocity, indicating that the smaller the particle size, the smaller is the interface velocity. For the typical size range $(0.2-5 \mu \mathrm{m})$ of ceramic powders, for example, the encapsulation speed typically falls within the speed range $(5-50 \mu \mathrm{m} / \mathrm{s})$ obtained under the usual processing conditions
[41]. Besides, the freezing temperature is also strongly dependent on the particle size, where the larger the particle size, the lower is the freezing temperature, which was thermodynamically demonstrated recently [30]. As a result, the lower the initial freezing temperature, the higher is the initial interface speed [56]. Thus, it is noted that particle size has a direct influence on the nucleation of ice/solid crystals in the suspension, since the smaller the particle size, the greater is the number of nucleation sites available and the lower the required supercooling.

\section{Solvent type}

In the freeze-casting, the type of solvent used to prepare the suspension is the main factor that affects the microstructure of the pores. Firstly, the solvent acts by dispersing the solid particles in the suspension. Then in the freezing step, it acts as a binder between the solid particles and also as a pore channel mold [45]. Although water is the most commonly used solvent, there is also much research and studies involving camphene, tert-butyl alcohol (TBA), and the naphthalene-camphor binary system. However, these solvents have different solidification temperatures, viscosities, surface tensions, and hydrophilicity, influencing the final macro and microstructure of the manufactured pieces $[35,45,68]$.

Water is generally used as the main solvent for various reasons, such as the convenience of use, environmental aspects, unique ice crystal morphologies that lead to unique porosities and compatibility with functional additives [56]. In water's particular case, the microstructure resulted after sublimation is lamellar, with lamellar channels between the ceramic walls [69]. However, due to its anisotropy, the lamellar structure of ice may have undesirable properties for some applications. Thus, alternatives are being sought to overcome this disadvantage. In this way, when the lamellar morphology is not desired, the camphene and tert-butyl alcohol (TBA) are some of the alternatives. Camphene $\left(\mathrm{C}_{10} \mathrm{H}_{16}\right)$ is a cyclic hydrocarbon, a crystalline plastic solid at room temperature, and also a natural and non-toxic material. Branched structures are typically obtained using camphene, thus forming dendritic-shaped pores when solidified under an appropriate temperature gradient [57]. Also, when the solid camphene is submitted to a heat treatment, large crystals are formed, which produce pieces with a macroporous microstructure [56]. Unlike the lamellar structure of frozen water or camphene, frozen TBA generally exhibits a long, straight prism crystal type, with no branching at its crystallization point, which is of great benefit for the manufacture of porous materials with membrane-like structures applied in the separation process $[37,70]$. TBA is often mixed with water as it can dissolve organic matter. The TBA/water mixtures are infinitely soluble but do not have a single form after solidification. The shape of the crystal varies with the ratio of TBA and water [68]. According to a study [68], the mixture of water and TBA solvents generates a wide variety of morphologies, ranging from lamellar to dendritic or acicular, 
as shown in Fig. 6. According to Tang et al. [68], in the initial additions of TBA in the TBA- $\mathrm{H}_{2} \mathrm{O}$ mixture, and by the freezing process, the first crystals have lamellar and dendritic structures. As the concentration of TBA increases in the TBA- $\mathrm{H}_{2} \mathrm{O}$ mixture, the crystals assume the following structures: snowflake, snowflake+dendritic, dendritic only, dendritic+needles, only needles, and lastly, there is only TBA in the suspension, and the crystals assume a hexagonal structure.

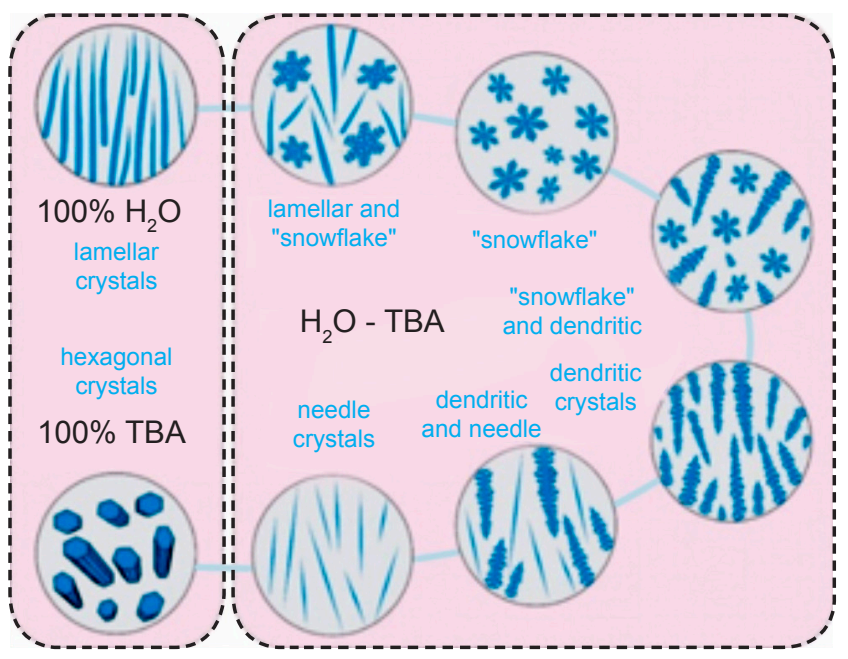

Figure 6: Morphology variation of pores in freeze-cast bodies according to the proportion of $\mathrm{H}_{2} \mathrm{O}$ and TBA.

Another solvent described in the literature is the binary compound naphthalene $\left(\mathrm{C}_{10} \mathrm{H}_{8}\right)$ and camphor $\left(\mathrm{C}_{10} \mathrm{H}_{16} \mathrm{O}\right)$. This binary compound has low viscosity, which allows preparing concentrated pastes to produce denser materials, and is, therefore, a promising solvent in the freeze-casting process for the manufacture of macroporous structures. Besides, the naphthalene-camphor solvent has a freezing retraction, leading to denser and more strong green bodies. The naphthalene-camphor solvent also has a relatively high melting temperature and vapor pressure, which allows freezing and sublime pastes under milder conditions. This behavior allows processing ceramic tiles under ambient

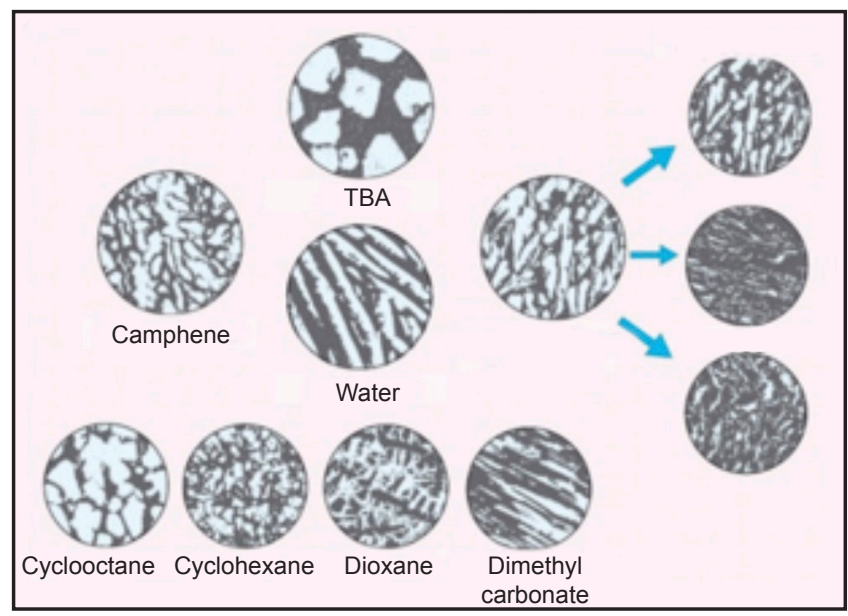

Figure 7: Types of microstructures obtained varying the solvent in the freeze-casting process. conditions using the freeze-casting method. However, this solvent may exhibit some toxicity $[71,72]$. Fig. 7 illustrates a schematic of the types of pore microstructures obtained when camphene, TBA, water, and naphthalene-camphor are employed as solvents to obtain ceramics pieces by the freeze-casting method. Still, in Fig. 7, it is possible to see a scheme of the pore microstructures when alternative solvents (cyclooctane, cyclohexane, dioxane, and dimethyl carbonate) are used [65]. However, alternative solvents can be incompatible with functional additives incorporated into the suspensions [56]; therefore, the most commonly used solvents are water, camphene, and TBA $[40,73]$.

\section{Temperature, rate, and direction of freezing}

During freezing of the suspension, the freezing temperature, rate, and time are process parameters that influence the nucleation and crystal growth of new solidphase, therefore, the morphology of porous microstructures $[50,74]$. In the freezing step of the freeze-casting method, the first experimental parameter to be adjusted is the temperature range used, which results in the freezing rate and temperature gradient. The suspension solidification can be unidirectional or not, and the temperature gradient can be fine-tuned, for example, with double-sided or multidirectional freezing configurations. In general, an increase in freezing temperature or the magnitude of the temperature gradient produces smaller crystals that turn into thinner pores [41]. The freezing temperature influences both the concentration of the nucleus and the crystal sizes. For example, when an aqueous solution is frozen into liquid nitrogen, the low temperature $\left(-196^{\circ} \mathrm{C}\right)$ results in a fast nucleation rate (i.e., several nuclei per volume unit) and small crystals. However, when the freezing step is accomplished at a higher temperature $\left(-20^{\circ} \mathrm{C}\right)$, the nucleation rate is slow, and large crystals are formed from them. Ceramic pieces manufactured under the latter experimental condition (low nucleation rate and larger crystals) show random and larger pores [42]. Fig. 8 shows the effect of the freezing temperature on the average pore size. Generally, the freezing temperature range employed on the freeze-casting method is between -18 to $-198^{\circ} \mathrm{C}[13,73]$.

For relatively high-temperature gradients, heat transfer is efficient, and solvent crystals can grow continuously, giving rise to interconnected or dendritic porous structures. On the other hand, when heat transfer is relatively low, it negatively affects the diffusion of solvent molecules, and therefore low crystal growth rates are observed. In the latter case, pores are more likely to have oval structures [78]. Also, the final microstructure is directly related to the shape and behavior of the solidification front, which can swallow or repel inert particles, so that by controlling the freezing direction and temperature gradient, aligned pore channels and a porous gradient can be achieved $[1,45]$. Changes in the morphology of the pores can be related to control of freezing direction through a process called directional freezing, which is characterized by the application of the temperature gradient on the suspension, 


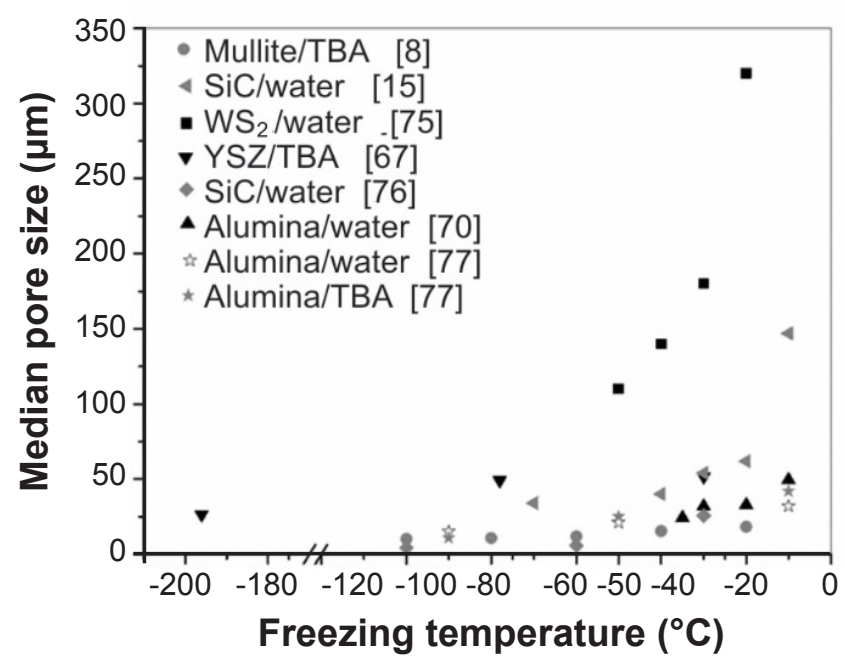

Figure 8: Relationship between freezing temperature and median pore size of ceramic materials obtained by freeze-casting $[8,15$, $67,70,75-77]$.

and as a consequence, crystals of the solvent first appear in the colder region and grow on the direction of the hotter region. The oriented crystals of the solvent can be removed by the sublimation process, and the resulting microstructure is composed of unidirectional pores $[42,79]$. When there is no temperature gradient in the suspension, the anisotropic growth is not observed, and a homogeneous microstructure is obtained [74]. Fig. 9 shows that the orientation of the ice crystals may be non-directional or present more than one orientation, depending on the freezing directions.

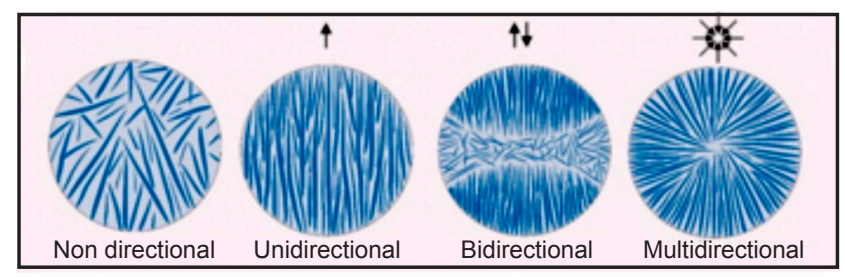

Figure 9: Crystal orientations using directional freezing.

Different freezing profiles lead to a change in the freeze front speed during the pouring of the suspension, which results in microstructures with different pore sizes [35]. Static freezing profiles are obtained when the colder side of the suspension is kept at a constant temperature and below the freezing temperature of the solvent throughout the freezecasting process. In the freezing step of the suspension, it is known that the morphology of the pores depends on the freezing conditions. A faster freezing front speed originates thinner crystals of the solvent. After the sublimation of the solvent, the channels contained in the microstructure is narrowed. Therefore, the control of the freezing front speed is equivalent to control the microstructure of the pores [35]. Also, during the solidification process of the suspension, the growth rate of the solvent crystals defines the freezing front speed, which determines whether a particle in the paste is rejected and 'pushed' forward or 'swallowed' and 'trapped' by the liquid-solid interface (Fig. 10). During freezing of the suspension, the rejection or entrapment of the particles depends on the critical speed of the freezing front. Porous microstructures are produced under conditions where the freezing front speed is less than the critical speed. Above the critical velocity, particles are entrapped by the solidification front, and the pores disappear from the final structure. However, if the freezing front speed is lower than the critical speed, the thickness of the crystal decreases with the rise of the solidification velocity [7]. Under this condition, lamellar and columnar geometries are produced because there is sufficient time for the rearrangement of the particles as they are trapped by the freezing front [38].

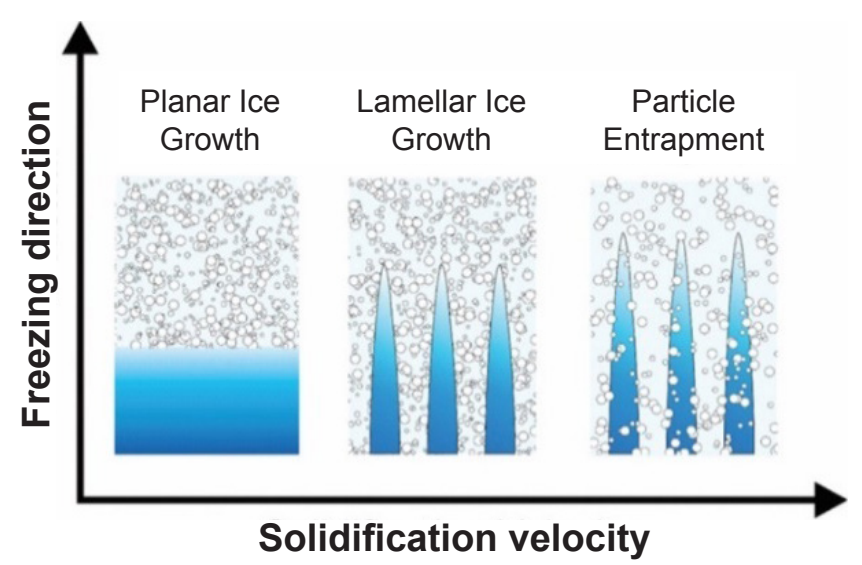

Figure 10: Influence of freezing speed on particle trapping.

The freezing front speed (Fig. 10) affects the number of particles involved, while for small particles, the number is not changed. When the freezing front grows rapidly, the particles are never pushed out of the freezing ice and thus remain homogeneously distributed. For cases in which the freezing front grows at an intermediate speed, particles diffuse over long distances, and the volume fraction in the interdendritic regions increases. If the freezing front velocity is low, the particles are pushed to form lamellar microstructures [78]. When the rate of solidification is extremely low, the lamellar structures may disappear. This behavior occurs because the freezing front speed is much lower than the critical particle trapping speed, and the low solidification speed makes the solidification front more stable. Thus, the solidification front has a flat morphology, in which all particles are 'discarded' together, and the directional microstructure cannot be obtained [78]. Fluctuations in the interface speed due to unbalanced conditions lead to variations in encapsulation behavior [41] and, consequently, the morphology of the microstructure. Several techniques are used to control pore size. The most common is a modification of the solidification rate. In general, fast and slow freezes result in smaller and larger pore sizes, respectively. However, this methodology is not convenient for obtaining large pores as very low freezing rates are required to obtain pores of a few hundred micrometers. In this way, other process parameters are modified, for example, with the use of additives [56]. 


\section{Additives}

The role of additives is probably the most promising and significantly in the solidification behavior, final microstructure of the pores, and mechanical behavior of the pieces manufactured by the freeze-casting method. Additives are added to modify the growth behavior of the crystals of solvents, which, subsequently, after sublimation, define pore morphology. It is well known that the crystal growth phenomenon is controlled by kinetic and thermodynamic factors. Thus, the addition of additives to the suspension affects the kinetic factors, decreasing or increasing the growth rate of the solvent crystals, and thermodynamic factors, decreasing surface free energy and changing the nucleation rate. Both affect the final morphology of the solvent crystals [41]. In addition to affecting the growth rate of the solvent crystals, the addition of additives into the suspension also changes the solvent phase diagram, anisotropy of the solid/ liquid interfacial energy, interparticle forces, degree of supercooling, viscosity of the solvent, freezing point of the suspension, and degree of expansion of the system. Also, some additives are capable of inducing gelation or binding with solvent molecules [53]. Additives already used in the literature include polyvinyl alcohol (PVA), glycerol, gelatin, sucrose, sodium chloride, or zirconium acetate [30, 68, 80]. In addition to the functions mentioned above, these additives are also used to control the stability and dispersion of suspensions, solidification behavior, and mechanical properties of the green body during and after sublimation [56]. Additives can change the microstructure morphology generating pores with different sizes and shapes. For example, manufactured pieces can change from lamellar morphology (e.g., without additive, with trehalose or sucrose) to cell morphology (e.g., gelatin, glycerol, or combination of sucrose with $\mathrm{pH}$ reduction using citric acid) or to a lamellar structure with a bimodal pore width distribution (e.g., using ethanol) [12,53]. Fig. 11 shows the additive's effect on some changes in the freezing morphology.

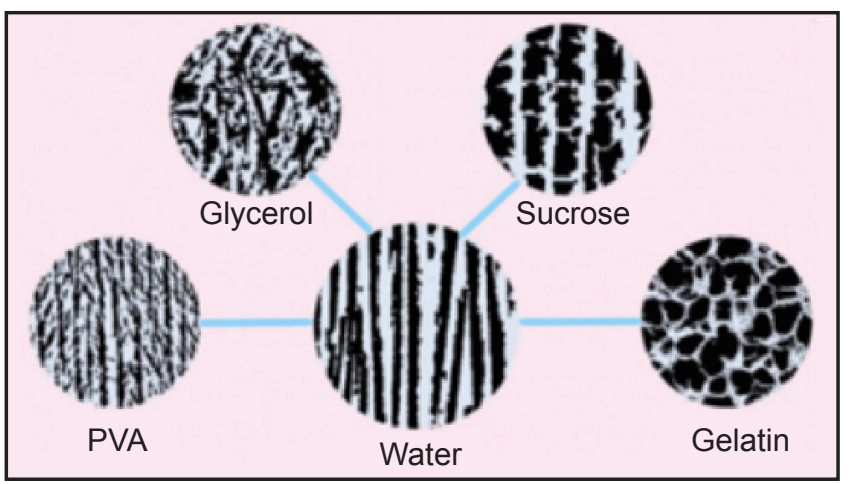

Figure 11: Microstructures obtained by the freeze-casting method using different additives.

Polyvinyl alcohol (PVA) is an organic binder used to strengthen the green body and also control the final pore size. According to White et al. [81], the increase in PVA content decreases pore size and favors the formation of dendritic structures with a higher degree of branching [82]. PVA is believed to favor the formation of dendritic crystals due to its antifreeze properties that lead to disturbances at the water-ice interface and higher supercooling [81]. Due to the PVA addition to the suspension, there is an increase in the viscosity of the suspension, and a gelling process at relatively high temperatures can occur. During the freezing step, the gelled PVA combines with solid particles, and the growth rate of the solvent crystals decreases. As a result, smaller size crystals arise from the solvent. Moreover, in cooling, the water solidifies, and the piece presents two phases (gelled PVA and solid water). Pore size and morphology depend on PVA concentration, and an increase in PVA amount results in a decrease in pore size [66, 83-85]. Still, about the PVA addition, large and isolated lamellar pores turn into small interconnected pores with lamellar or columnar structures [83-85]. Glycerol is miscible in water, and when added to the suspension, immediately modifies the local structure of the aqueous liquid. As a result of the addition of glycerol, there is a change in the nucleation and growth rates of ice crystals, thus modifying the freezing behavior of the aqueous liquid [74]. As a cryoprotectant, glycerol lowers the freezing temperature of the liquid solution and binds to water molecules, making ice crystallization difficult. This leads to a reduction in the size of ice crystals. It also reduces water volume expansion in solidification, reducing associated defects [54, 74, 82]. Although glycerol improves the homogeneity of the microstructure, the amount added to the suspension should be monitored because glycerol has low vapor pressure. After freeze-casting, glycerol remains in the manufactured part and generally needs to be removed. Excess glycerol increases the viscosity of the suspension or prevents sample drying even after sublimation of the ice. A wet sample, due to the excessive presence of glycerol, may lead to the deformation of the shape [54]. Finally, the excess glycerol in a suspension processed via the freeze-casting method results in pieces with lower porosity due to the lower ice expansion during the freezing process [78].

Modifying the chemical composition of the solvent, and therefore, its physical properties is another way to control pore morphology in the freeze casting method. Adding gelling agents to the suspension before the freezing step is a way of controlling pore morphology [35]. The use of gelatin can modify pores with directional morphology to homogeneous pores with cellular or spherical microstructure. Fukushima's research group [2, 14-19, 21] accomplish studies that combined the freeze-casting method with the use of gelatin. They showed that the prior gelation of the suspension completely alters the pore morphology of the manufactured pieces. Cilla et al. [69] also studied how the use of gelatin influences the morphology of the pores without prior gelation. The authors argued that firstly the formation of micelles occurs when the critical micellar concentration (CMC) is achieved. These micelles have a critical size and act as nuclei, favoring the nucleation of 
ice crystals with subsequent formation of homogeneous pore morphology. Published studies [80, 81] showed that the addition of zirconium acetate $(\mathrm{ZrAc})$ to the suspension changed the lamellar pore structure to a columnar structure. Besides, the presence of a zirconium acetate complex in the suspension results in a honeycomb-like pore morphology [80]. The addition of sucrose changed the structure of the snowflake or dendritic pores to a small cylindrical structure [24]. When an $\mathrm{H}_{2} \mathrm{O}$-TBA mixture was used as a solvent, the authors reported that sucrose can be dissolved in water but is insoluble in TBA. However, sucrose molecules in the suspension containing water and TBA could inhibit the crystal growth of ice and TBA during directional freezing. According to Munch et al. [53], different additives added for ceramic suspensions changed the structure of the ice crystals, and consequently, the final morphology of the manufactured piece. In agreement with these authors, the addition of 10 wt $\%$ sucrose in citrus water with $\mathrm{pH} 2.5$ results in a cellular structure with very smooth ceramic walls; the addition of 4 $\mathrm{wt} \%$ of ethanol results in a rough lamellar structure; $4 \mathrm{wt} \%$ of sodium chloride addition results in a sharp-sided lamellar surface; and $4 \mathrm{wt} \%$ of sucrose addition promotes a lamellar structure with microscopic roughness $[53,86]$.

Other additives can also be added to the suspension processed by the freeze-casting method. An example is the use of monofunctional alcohols such as ethanol $\left(\mathrm{C}_{2} \mathrm{H}_{5} \mathrm{OH}\right)$, propanol $\left(\mathrm{C}_{3} \mathrm{H}_{7} \mathrm{OH}\right)$, butanol $\left(\mathrm{C}_{4} \mathrm{H}_{9} \mathrm{OH}\right)$, methanol $\left(\mathrm{CH}_{3} \mathrm{OH}\right)$, and isopropyl alcohol (IPA), which results in a dramatical increase of the pore size. This is because alcohols induce the formation of clathrate hydrates, which are nonstoichiometric structures, where an alcohol molecule is trapped in a hydrogen-bonded network (such as water), and this structure leads to an increase in pore size. Also, studies $[87,88]$ have shown that the addition of alcohols such as ethanol and propanol can increase the viscosity of the suspension, inhibit the expulsion of ceramic particles from the ice crystals, and cause a slower growth rate of ice crystals. The addition of IPA resulted in elongated ice crystals perpendicular to the direction of ice growth $[30,89]$. Although different types of additives such as polyvinyl alcohol (PVA), zirconium acetate, and sucrose all influence the speed of the freezing front, and therefore the microstructure of manufactured parts, these additives cannot be directly removed by sublimation and always require a calcination process or pre-sintering treatment [37], which also causes the formation of pores with the burning of these organics. Thus, these additives also act as classic porogenic agents, and according to the additive content incorporated into the suspension, some care with their elimination should be taken during sintering.

\section{MECHANICAL PROPERTIES}

The control of pore size and morphology is generally the critical factor that significantly affects the final properties, and consequently, the applications of porous ceramics [50]. In this way, all factors that influence the modification of the quantity, size, and morphology of the pores, which have been discussed previously, determine the properties of the final piece. As predicted, the higher the porosity, the lower is the mechanical resistance of the piece manufactured since the pores act as stress concentrators, assisting in the propagation of cracks when subjected to mechanical stresses. Generally, the compressive strength is the leading mechanical property measured in the investigations already published [56]. Fig. 12 shows the correlation between the degree of porosity and the compressive strength of several studies, making it evident that these factors are inversely proportional.

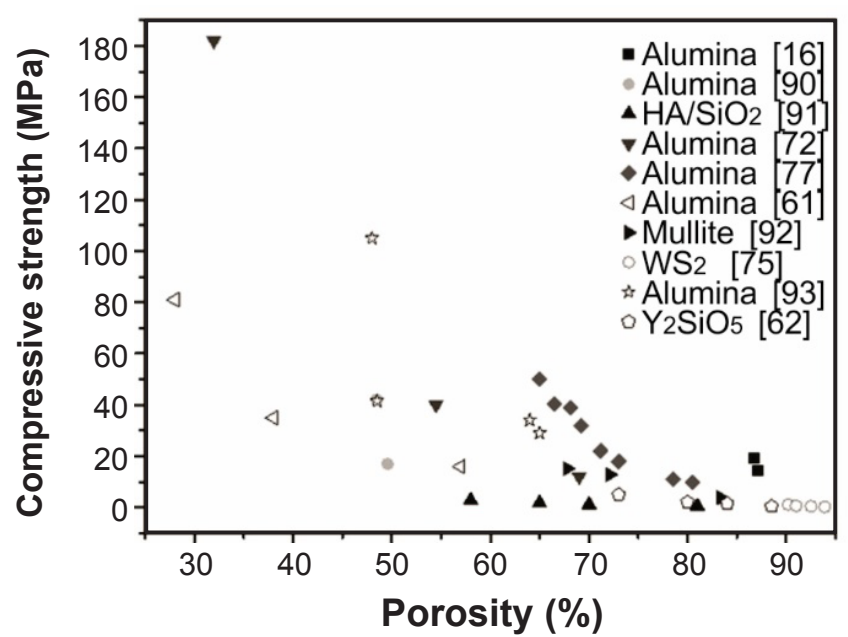

Figure 12: Correlation between porosity and compressive strength of ceramics manufactured by freeze-casting $[16,61,62,72,75$, 77, 90-93].

It is worth mentioning that an in-depth study on the mechanical properties of ceramics obtained by freezecasting has not yet been found in the literature, as these values may differ widely depending on the nature and amount of ceramic powder used, solvent, use of additives, pore quantity, shape, and size, among other characteristics that affect the final mechanical properties. For example, the particle size has an important effect on the mechanical properties since powders with a smaller particle size improve the densification of the ceramic resulting in a material with less porosity [50]. Also, the increase in the solid load on the suspension can promote the improvement of the mechanical properties. Another critical factor is the geometry of the pores since one of the main characteristics of the freezecasting method is to obtain lamellar/dendritic pores, and this type of pore results in ceramics with anisotropic properties showing different strength values depending on the direction of the applied load. Also, the use of additives can be an artifice to try to improve the mechanical resistance of the pieces. For example, the interaction between glycerol and other components in the pastes promotes a good connection between the lamellas and, thus, improves the mechanical properties of porous ceramics [50].

Given the low mechanical resistance achieved due to the high porosity, some authors try to overcome this disadvantage by including new process steps. Among these steps, stand 
out the use of a fifth post-processing step, which consists of an infiltration of a second phase (polymeric, metallic, or glassy) to form a two-phase composite and, therefore, result in increased mechanical properties that exceed a simple mixture of its constituents. Also, the application of magnetic fields during some process steps has been shown to improve the mechanical properties of the piece manufactured. This is a consequence of the particle alignment on the direction of the magnetic field applied [30]. Although these post-processing techniques have provided excellent results, accurate control of the freeze-casting process is the result of the freezing step, as this is where the porosity and properties of ceramics are initially developed [30]. Furthermore, studies still need to be carried out to find a way to improve the strength of ceramics obtained by freeze-casting while maintaining high porosity.

\section{APPLICATIONS}

The freeze-casting method is becoming increasingly used in many applications due to the numerous possibilities of pore morphological control. Among these applications, stand out scaffolds, solid oxide fuel cells, hot gas separation membrane, thermal insulators, etc. [48, 94].

Scaffolds: are widely used in bone tissue regeneration, providing a support structure for cell colonization, proliferation, and subsequent new bone formation. Among the many characteristics that an ideal scaffold should have, stand out mechanical properties compatible with the tissue to be regenerated, highly porous structure, excellent bioactivity, and good biodegradability [95]. The freezecasting method is already applied in the production of scaffolds from synthetic and natural polymers $[60,75$, 77, 91, 95-97], and recently, its application to obtain bioceramic scaffolds [74] is receiving great scientific and technological interest. To allow cells to grow through the scaffold, thus allowing their integration with the tissue to be regenerated, high concentrations of pores, large surface area, adequate pore size (between 50 to $200 \mu \mathrm{m}$ ), and a highly interconnected pore network are required, besides the already mentioned biocompatibility and biodegradability [96]. Scaffolds synthesized from hydroxyapatite are good examples of these types of materials manufactured using the freeze-casting method [23, 74, 91, 95, 98]. These scaffolds show excellent mechanical properties, even with high open porosity, and are potential candidates for biomaterials such as tooth and bone implants. For dental applications, scaffolds with high porosities and small pore sizes are required. Thus, the freeze-casting method is particularly interesting because the manufactured pieces present pore sizes on the micrometer scale. It is already possible to find works in the literature in which the porous hydroxyapatite scaffolds were designed to replace the dentin [23]. These scaffolds are interesting because hydroxyapatite (HA) and dentin have similar chemical composition, and pore size between 3 and $5 \mu \mathrm{m}$ in the manufactured parts allows dentin permeability. Porous hydroxyapatite scaffolds obtained by the freezecasting method are competitive in the market, reproducible, clinically relevant, and biocompatible with the dentin [23]. Water is an environmentally friendly solvent, and the use of ice to obtain porous ceramics pieces by the freeze-casting method is green and sustainable. Because of this, scaffolds manufactured by the freeze-casting method using water as a solvent has gained high visibility in recent years in tissue engineering [42]. However, other solvents are used for the production of scaffolds. Singh and Soundarapandian [60] adopted the freeze-casting method, in which camphene was the solvent, to obtain scaffolds with micro-sized particles. By applying different freezing conditions and solid fillers content, it was possible to control pore size, porosity, and compressive strength. These authors pointed out that the freeze-casting method was efficient for the production of bone scaffolds.

Energy applications: materials used in the energy applications area, such as solid oxide fuel cells, hot gas separation membrane, catalytic membrane reactor, and molten metal filters, also can be manufactured from the freeze-casting method $[3,4,40]$. Ceramic membranes are widely used in energy applications, and the freeze-casting method can be used to produce mono and bi-layers ceramic membranes. In single-layer membranes, the microstructure has to be controlled to avoid the presence of asymmetric pores, which is achieved by controlling the freezing speed and using additives. Those consisting of bi- or multi-layers contain a thin layer, which accounts for the filtration/ treatment itself, and the other layers are the substrate that provides mechanical resistance for the first. The need for this substrate layer is that thin ceramic layers are fragile and have low strength. High permeability substrates are required in bi- or multi-layers membranes, which can be achieved with an asymmetric structure or oriented pores. The use of substrates consisting of pores with asymmetric structure allows to orient and maximize the flow of the filtrate material. Moreover, the use of aligned pore substrates with columnar characteristics allows adequate mechanical resistance to the thinner layer and increases the robustness of the membranes [99]. According to Souza et al. [100, 101], who studied the influence of process parameters on the permeability of asymmetric ceramic membranes obtained by freeze-casting, it is evident that the greater the open porosity with an interconnected pore network, the greater the gas permeability. In this way, the freeze-casting method, with appropriate control of the process, makes it possible to obtain ceramic parts with pore asymmetry and oriented pore network. The freeze-casting method can also be used to obtain linear, unidirectional foams. When camphene or water is used as a freezing vehicle, dendritic structures are formed along the aligned channels. These dendritic structures connect the channels improving flow properties $[3,67]$. These foams can have the diameter of the channels adjusted by the temperature gradient, freezing velocity, or additives used and have wide flexibility of applications.

Thermal insulation: thermal conductivity is an intrinsic property; however, it is unquestionable that the concentration and morphology of the pore also significantly increase the 
insulating characteristics of various types of materials. It is essential to emphasize that to develop porous ceramics with a specific thermal conductivity, the processing method, as well as its process parameters, should be carefully chosen [102]. A common feature of microstructures of pieces produced by freeze-casting is a dendritic structure. The presence of dendrites promotes interconnectivity between the pore channels and the cross-flow convection between the channels. These characteristics must be avoided for insulation applications. However, microstructures with pore channels without dendritic characteristics can be obtained using freeze-casting when appropriate additives and/or solvents are used [3]. Fukushima et al. [15, 20, 21] used gelling agents to the freeze-casting method to obtain porous ceramics with suitable pore structures for thermal insulation. The ideal pore structure for insulating materials is formed by vertical and parallel pores or spherical isolated pores. Vertical and parallel pores were achieved in this researches. Other researchers [102] used TBA solvent to prevent dendrite formation, and vertical and parallel pores were also produced. Thus, the freeze-casting method, according to the appropriate use of the solvent/additive combination, has the potential for the production of porous materials for thermal insulation applications.

\section{CONCLUSIONS}

The freeze-casting method shows great versatility in the production of porous materials, which makes it very attractive for a variety of applications. Water is the solvent typically used in this process and generates directional and aligned pores. Recently, studies have a focus on the use of additives for the modification of the pore microstructure produced when water is the solvent. Scientific works published in the last decade also addressed the use of other solvents to obtain non-dendritic pore microstructures or aligned vertical pores. The solid amount, freezing conditions, and type of additives are the more important parameters in the control of the pore morphology of freeze-cast pieces. The freeze-casting process has the potential to cover applications that require porous materials with various morphologies, from lamellar to cellular, as well as pore size materials ranging from micro to macropores.

\section{ACKNOWLEDGMENTS}

This study was financed in part by the Coordenação de Aperfeiçoamento de Pessoal de Nível Superior - Brasil (CAPES) - Finance Code 001 (scholarship granted to Mrs. Larrisea Nathiere Rangel de Morais Santos). The authors are grateful to the Brazilian research funding agency CNPq, grant nos. 308822/2018-8 and 420004/2018-1, for the financial support.

\section{REFERENCES}

[1] S. Deville, E. Saiz, A.P. Tomsia, Acta Mater. 55, 6 (2007)
1965.

[2] M. Fukushima, Y.-I. Yoshizawa, T. Ohji, Adv. Eng. Mater. 16, 6 (2014) 607.

[3] E.C. Hammel, O.L.R. Ighodaro, O.I. Okoli, Ceram. Int. 40, 10 (2014) 15351.

[4] R.K. Nishihora, P.L. Rachadel, M.G.N. Quadri, D. Hotza, J. Eur. Ceram. Soc. 38, 4 (2018) 988.

[5] T. Ohji, M. Fukushima, Int. Mater. Rev. 57, 2 (2013) 115.

[6] A.R. Studart, U.T. Gonzenbach, E. Tervoort, L.J. Gauckler, J. Am. Ceram. Soc. 89, 6 (2006) 1771.

[7] S. Deville, Adv. Eng. Mater. 10, 3 (2008) 155.

[8] R. Liu, J. Yuan, C.-A. Wang, J. Eur. Ceram. Soc. 33, 1516 (2013) 3249.

[9] W.A. Maxwell, R.S. Gurnick, A.C. Francisco, "Preliminary investigation of the 'freeze-casting' method for forming refractory powders", Nat. Advis. Comm. Aeronaut., Washington (1954).

[10] H. Tong, C. Gryte, Colloid Polym. Sci. 263, 2 (1985) 147.

[11] H.-M. Tong, I. Noda, C.C. Gryte, Colloid Polym. Sci. 262, 7 (1984) 589.

[12] T. Fukasawa, M. Ando, T. Ohji, S. Kanzaki, J. Am. Ceram. Soc. 84, 1 (2001) 230.

[13] T. Fukasawa, Z.-Y. Deng, M. Ando, T. Ohji, Y. Goto, J. Mater. Sci. 36, 10 (2001) 2523.

[14] M. Fukushima, H. Hyuga, C. Matsunaga, Y.-I. Yoshizawa, J. Am. Ceram. Soc. 101, 8 (2018) 3266.

[15] M. Fukushima, M. Nakata, Y. Zhou, T. Ohji, Y.-I. Yoshizawa, J. Eur. Ceram. Soc. 30, 14 (2010) 2889.

[16] M. Fukushima, T. Ohji, H. Hyuga, C. Matsunaga, Y.-I. Yoshizawa, J. Mater. Res. 32, 17 (2017) 3286.

[17] M. Fukushima, S. Tsuda, Y.-I. Yoshizawa, L. Gauckler, J. Am. Ceram. Soc. 96, 4 (2013) 1029.

[18] M. Fukushima, Y.-I. Yoshizawa, Mater. Lett. 153 (2015) 99.

[19] M. Fukushima, Y.-I. Yoshizawa, Adv. Powder Technol. 27, 3 (2016) 908.

[20] M. Fukushima, Y.-I. Yoshizawa, J. Eur. Ceram. Soc. 36, 12 (2016) 2947.

[21] M. Fukushima, Y.-I. Yoshizawa, P. Colombo, J. Am. Ceram. Soc. 97, 3 (2014) 713.

[22] Y. Tang, C. Wu, K. Zhao, Ceram. Int. 44, 5 (2018) 5794.

[23] Y. Tang, M. Mao, R. Xu, K. Zhao, Z. Duan, Ceram. Int. 44, 10 (2018) 11883.

[24] Y. Tang, M. Mao, S. Qiu, K. Zhao, W. Chen, Ceram. Int. 44, 14 (2018) 17566.

[25] Y. Tang, M. Mao, S. Qiu, K. Zhao, Ceram. Int. 44, 1 (2018) 1187.

[26] Y. Tang, M. Mao, S. Qiu, C. Wu, J. Eur. Ceram. Soc. 38, 11 (2018) 4149.

[27] S. Devavarapu, P. Chaudhuri, A. Shrivastava, S. Bhattacharyya, Ceram. Int. 45, 9 (2019) 12264.

[28] N. Guo, P. Shen, R.-F. Guo, Q.-C. Jiang, Mater. Sci. Eng. A 748 (2019) 286.

[29] K. Hong, H. Park, Y. Kim, M. Knapek, P. Minarik, K. Mathis, A. Yamamoto, H. Choe, J. Mech. Behav. Biomed. Mater. 98 (2019) 213. 
[30] I. Nelson, S.E. Naleway, J. Mater. Res. Technol. 8, 2 (2019) 2372.

[31] M. Rashad, U. Sabu, G. Logesh, M. Balasubramanian, Sep. Purif. Technol. 219 (2019) 74.

[32] N. Wang, Y. Liu, Y. Zhang, Y. Du, J. Zhang, Ceram. Int. 45, 9 (2019) 11558.

[33] M. Zhang, X. Li, M. Zhang, Z. Xiu, J.-G. Li, J. Li, M. Xie, J. Chen, X. Sun, J. Mater. Sci. 54, 14 (2019) 10119.

[34] T. Zheng, J.-J. Li, L.-L. Wang, Z.-J. Wang, J.-C. Wang, Int. J. Heat Mass Transf. 133 (2019) 986.

[35] C.D. Christiansen, K.K. Nielsen, R.K. Bordia, R. Bjørk, J. Am. Ceram. Soc. 102, 10 (2019) 5796.

[36] S. Christoph, A. Hamraoui, E. Bonnin, C. Garnier, T. Coradin, F.M. Fernandes, Chem. Eng. J. 350, (2018) 20.

[37] S. Dong, L. Wang, X. Gao, W. Zhu, Z. Wang, Z. Ma, C. Gao, J. Membr. Sci. 541 (2017) 143.

[38] S.A. Barr, E. Luijten, Acta Mater. 58, 2 (2010) 709.

[39] A.A. Al-Attar, M. Asle Zaeem, S.A. Ajeel, N.E.A. Latiff, J. Eur. Ceram. Soc. 37, 4 (2017) 1635.

[40] C. Gaudillere, J.M. Serra, Bol. Soc. Esp. Ceram. V. 55, 2 (2016) 45.

[41] S. Deville, J. Mater. Res. 28, 17 (2013) 2202.

[42] L. Qian, H. Zhang, J. Chem. Technol. Biotechnol. 86, 2 (2011) 172.

[43] M.C. Gutiérrez, M.L. Ferrer, F. del Monte, Chem. Mater. 20, 3 (2008) 634.

[44] S. Deville, E. Saiz, R.K. Nalla, A.P. Tomsia, Science 311, 5760 (2006) 515.

[45] R. Chen, C.-A. Wang, Y. Huang, L. Ma, W. Lin, J. Am. Ceram. Soc. 90, 11 (2007) 3478.

[46] S. Deville, E. Maire, A. Lasalle, A. Bogner, C. Gauthier, J. Leloup, C. Guizard, J. Am. Ceram. Soc.93, 9 (2010) 2507. [47] K. Lu, C.S. Kessler, R.M. Davis, J. Am. Ceram. Soc. 89, 8 (2006) 2459.

[48] J. Han, L. Hu, Y. Zhang, Y. Zhou, J. Am. Ceram. Soc. 92, 9 (2009) 2165.

[49] Y. Chino, D.C. Dunand, Acta Mater. 56, 1 (2008) 105.

[50] R. Liu, T. Xu, C.-A. Wang, Ceram. Int. 42, 2 (2016) 2907.

[51] S.R. Mukai, H. Nishihara, H. Tamon, Chem. Commun. 7 (2004) 874.

[52] H. Nishihara, S.R. Mukai, D. Yamashita, H. Tamon, Chem. Mater. 17, 3 (2005) 683.

[53] E. Munch, E. Saiz, A.P. Tomsia, S. Deville, J. Am. Ceram. Soc. 92, 7 (2009) 1534.

[54] S.W. Sofie, F. Dogan, J. Am. Ceram. Soc. 84, 7 (2001) 1459.

[55] W. Li, K. Lu, J.Y. Walz, J. Am. Ceram. Soc. 96, 6 (2013) 1763.

[56] S. Deville, Materials 3, 3 (2010) 1913.

[57] K. Araki, J.W. Halloran, J. Am. Ceram. Soc. 88, 5 (2005) 1108.

[58] Z. Hou, B. Zhang, R. Zhang, L. Liu, Ceram. Int. 43, 12 (2017) 8809.

[59] L. Li, Q. Li, J. Hong, M. Sun, J. Zhang, S. Dong, J. Alloys Compd. 732 (2018) 136.

[60] G. Singh, S. Soundarapandian, J. Mech. Behav. Biomed.
Mater. 79 (2018) 189.

[61] Y. Tang, Q. Miao, S. Qiu, K. Zhao, L. Hu, J. Eur. Ceram.

Soc. 34, 15 (2014) 4077.

[62] R. Zhang, Q. Qu, B. Han, B. Wang, Mater. Lett. 175 (2016) 219.

[63] R. Asthana, S. Tewari, J. Mater. Sci. 28, 20 (1993) 5414. [64] S.M. Miller, X. Xiao, J.A. Setlock, K.T. Faber, J. Eur. Ceram. Soc. 38, 15 (2018) 5134.

[65] M. Naviroj, P.W. Voorhees, K.T. Faber, J. Mater. Res. 32, 17 (2017) 3372.

[66] C. Peko, B. Groth, I. Nettleship, J. Am. Ceram. Soc. 93, 1 (2010) 115 .

[67] L. Hu, C.-A. Wang, Y. Huang, C. Sun, S. Lu, Z. Hu, J. Eur. Ceram. Soc. 30, 16 (2010) 3389.

[68] Y. Tang, S. Qiu, C. Wu, Q. Miao, K. Zhao, J. Eur. Ceram. Soc. 36, 6 (2016) 1513.

[69] M.S. Cilla, C.J. Melo, R.R. Menezes, S.L.N. Lima, N.G. Araujo, Ceram. Int. 42, 7 (2016) 9278.

[70] S.M. Miller, X. Xiao, K.T. Faber, J. Eur. Ceram. Soc. 35, 13 (2015) 3595.

[71] K. Araki, J.W. Halloran, J. Am. Ceram. Soc. 87, 11 (2004) 2014.

[72] L.D. Lacerda, D.F. Souza, E.H.M. Nunes, M. Houmard, Ceram. Int. 44, 13 (2018) 16010.

[73] T. Wu, W. Zhang, B. Yu, J. Chen, Int. J. Hydrog. Energy 42, 50 (2017) 29900.

[74] Q. Fu, M.N. Rahaman, F. Dogan, B.S. Bal, J. Biomed. Mater. Res. B Appl. Biomater. 86, 1 (2008) 125.

[75] J. Wu, B. Luo, X. Liu, L. Zhang, J. Porous Mater. 25, 1 (2017) 37.

[76] V. Naglieri, P. Colombo, J. Eur. Ceram. Soc. 37, 7 (2017) 2559.

[77] M.-Q. Sun, P. Shen, Q.-C. Jiang, J. Mater. Sci. 54, 7 (2018) 5224.

[78] W.L. Li, K. Lu, J.Y. Walz, Int. Mater. Rev. 57, 1 (2013) 37.

[79] H. Zhang, A.I. Cooper, Adv. Mater. 19, 11 (2007) 1529.

[80] S. Deville, C. Viazzi, J. Leloup, A. Lasalle, C. Guizard, E. Maire, J. Adrien, L. Gremillard, PLoS One 6, 10 (2011) e26474.

[81] M.A. White, J. Conrad, S.N. Ellis, R. Chen, J. Am. Ceram. Soc. 100, 11 (2017) 5066.

[82] K. Lebreton, J.M. Rodríguez-Parra, R. Moreno, M.I. Nieto, Adv. Appl. Ceram. 114, 5 (2015) 296.

[83] L. Ren, Y.-P. Zeng, D. Jiang, Ceram. Int. 35, 3 (2009) 1267.

[84] K.H. Zuo, Y.P. Zeng, D. Jiang, Int. J. Appl. Ceram. Technol. 5, 2 (2008) 198.

[85] K.H. Zuo, Y.P. Zeng, D. Jiang, Mater. Sci. Eng. C 30, 2 (2010) 283.

[86] J. Roleček, D. Salamon, Z. Chlup, J. Eur. Ceram. Soc. 37, 14 (2017) 4279.

[87] M.M. Porter, R. Imperio, M. Wen, M.A. Meyers, J. McKittrick, Adv. Funct. Mater. 24, 14 (2014) 1978.

[88] J. Zeng, Y. Zhang, K. Zhou, D. Zhang, Trans. Nonferr. Met. Soc. China 24 (2014) 718.

[89] S. Miller, X. Xiao, J. Setlock, S. Farmer, K. Faber, Int. 
J. Appl. Ceram. Technol. 15, 2 (2018) 296.

[90] Z.-J. Hu, X.-T. Shen, S.-L. Geng, P. Shen, Q.-C. Jiang, Ceram. Int. 44, 5 (2018) 5803.

[91] Z.-Q. Jia, Z.-X. Guo, F. Chen, J.-J. Li, L. Zhao, L. Zhang, Ceram. Int. 44, 4 (2018) 3636.

[92] Z. Wang, P. Feng, X. Wang, P. Geng, F. Akhtar, H. Zhang, Ceram. Int. 42, 10 (2016) 12414.

[93] Y. Zhang, L. Hu, J. Han, Z. Jiang, Ceram. Int. 36, 2 (2010) 617.

[94] D.F. Souza, E.H.M. Nunes, J.A. Queiroga, W.L. Vasconcelos, J. Eur. Ceram. Soc. 38, 11 (2018) 4020.

[95] H. Semyari, M. Salehi, F. Taleghani, A. Ehterami, F. Bastami, T. Jalayer, H. Semyari, M. Hamed Nabavi, H. Semyari, J. Biomater. Appl. 33, 4 (2018) 501.

[96] M.-H. Ho, P.-Y. Kuo, H.-J. Hsieh, T.-Y. Hsien, L.-T.
Hou, J.-Y. Lai, D.-M. Wang, Biomaterials 25, 1 (2004) 129. [97] L. Yan, J. Wu, L. Zhang, X. Liu, K. Zhou, B. Su, Mater. Sci. Eng. C 75 (2017) 335.

[98] W. Guo, J. Liu, P. Zhang, L. Song, X. Wang, Y. Hu, Compos. Sci. Technol. 158 (2018) 128.

[99] P.L. Rachadel, D.F. Souza, E.H.M. Nunes, J.C.D. da Costa, W.L. Vasconcelos, D. Hotza, J. Eur. Ceram. Soc. 37, 16 (2017) 5249.

[100] D.F. Souza, E.H.M. Nunes, W.L. Vasconcelos, Ceram. Int. 44, 1 (2018) 1002.

[101] D.F. Souza, E.H.M. Nunes, D.S. Pimenta, D.C.L. Vasconcelos, J.F. Nascimento, W. Grava, M. Houmard, W.L. Vasconcelos, Mater. Charact. 96 (2014) 183.

[102] D. Li, M. Li, J. Mater. Sci. Technol. 28, 9 (2012) 799. (Rec.06/04/2020, Rev. 04/06/2020, Ac. 29/06/2020) 